\title{
INVESTIGATING THE COSMIC-RAY IONIZATION RATE NEAR THE SUPERNOVA REMNANT IC 443 THROUGH $\mathrm{H}_{3}^{+}$OBSERVATIONS ${ }^{*} \dagger$
}

\author{
Nick Indriolo ${ }^{1}$, Geoffrey A. Blake ${ }^{2}$, Miwa Goto ${ }^{3}$, Tomonori Usuda ${ }^{4}$, TaKeshi OKa ${ }^{5}$, T. R. Geballe ${ }^{6}$, Brian D. Fields ${ }^{1,7}$, \\ AND BENJAMIN J. MCCALL $1,7,8$ \\ ${ }^{1}$ Department of Astronomy, University of Illinois at Urbana-Champaign, Urbana, IL 61801, USA \\ ${ }^{2}$ Division of Geological and Planetary Sciences and Division of Chemistry and Chemical Engineering, MS 150-21, California Institute of Technology, \\ Pasadena, CA 91125, USA \\ ${ }^{3}$ Max-Planck-Institut für Astronomie, Königstuhl 17, Heidelberg D-69117, Germany \\ ${ }^{4}$ Subaru Telescope, 650 North A'ohoku Place, Hilo, HI 96720, USA \\ ${ }^{5}$ Department of Astronomy and Astrophysics and Department of Chemistry, University of Chicago, Chicago, IL 60637, USA \\ ${ }^{6}$ Gemini Observatory, 670 North A'ohoku Place, Hilo, HI 96720, USA \\ ${ }^{7}$ Department of Physics, University of Illinois at Urbana-Champaign, Urbana, IL 61801, USA \\ ${ }^{8}$ Department of Chemistry, University of Illinois at Urbana-Champaign, Urbana, IL 61801, USA \\ Received 2010 July 7; accepted 2010 September 24; published 2010 November 12
}

\begin{abstract}
Observational and theoretical evidence suggests that high-energy Galactic cosmic rays are primarily accelerated by supernova remnants. If also true for low-energy cosmic rays, the ionization rate near a supernova remnant should be higher than in the general Galactic interstellar medium (ISM). We have searched for $\mathrm{H}_{3}^{+}$absorption features in six sight lines which pass through molecular material near IC 443-a well-studied case of a supernova remnant interacting with its surrounding molecular material-for the purpose of inferring the cosmic-ray ionization rate in the region. In two of the sight lines (toward ALS 8828 and HD 254577) we find large $\mathrm{H}_{3}^{+}$column densities, $N\left(\mathrm{H}_{3}^{+}\right) \approx 3 \times 10^{14} \mathrm{~cm}^{-2}$, and deduce ionization rates of $\zeta_{2} \approx 2 \times 10^{-15} \mathrm{~s}^{-1}$, about five times larger than inferred toward average diffuse molecular cloud sight lines. However, the $3 \sigma$ upper limits found for the other four sight lines are consistent with typical Galactic values. This wide range of ionization rates is likely the result of particle acceleration and propagation effects, which predict that the cosmic-ray spectrum and thus ionization rate should vary in and around the remnant. While we cannot determine if the $\mathrm{H}_{3}^{+}$absorption arises in post-shock (interior) or pre-shock (exterior) gas, the large inferred ionization rates suggest that IC 443 is in fact accelerating a large population of low-energy cosmic rays. Still, it is unclear whether this population can propagate far enough into the ISM to account for the ionization rate inferred in diffuse Galactic sight lines.
\end{abstract}

Key words: astrochemistry - cosmic rays - ISM: supernova remnants

\section{INTRODUCTION}

As cosmic rays propagate through the interstellar medium (ISM), they interact with the ambient material. These interactions include excitation and ionization of atoms and molecules, spallation of nuclei, excitation of nuclear states, and the production of neutral pions $\left(\pi^{0}\right)$ which decay into gamma rays. Evidence suggests that Galactic cosmic rays are primarily accelerated by supernova remnants (SNRs) through the process of diffusive shock acceleration (e.g., Drury 1983; Blandford \& Eichler 1987), so interstellar clouds in close proximity to an SNR should provide a prime "laboratory" for studying these interactions. IC 443 represents such a case, as portions of the SNR shock are known to be interacting with the neighboring molecular clouds.

IC 443 is an intermediate age remnant (about 30,000 yr; Chevalier 1999) located in the Galactic anti-center region $(l, b) \approx\left(189^{\circ},+3^{\circ}\right)$ at a distance of about $1.5 \mathrm{kpc}$ in the Gem OB1 association (Welsh \& Sallmen 2003), and is a particularly well-studied SNR. Figure 1 shows the red image of IC 443 taken during the Second Palomar Observatory Sky Survey

\footnotetext{
* Some of the data presented herein were obtained at the W. M. Keck Observatory, which is operated as a scientific partnership among the California Institute of Technology, the University of California and the National Aeronautics and Space Administration. The Observatory was made possible by the generous financial support of the W. M. Keck Foundation.

$\dagger$ Based in part on data collected at Subaru Telescope, which is operated by the National Astronomical Observatory of Japan.
}

(POSS-II). The remnant is composed of subshells A and B; shell $\mathrm{A}$ is to the northeast-its center at $\alpha=06^{\mathrm{h}} 17^{\mathrm{m}} 08^{\mathrm{s}} .4$, $\delta=+22^{\circ} 36^{\prime} 39^{\prime \prime} .4 \mathrm{~J} 2000.0$ is marked by the cross-while shell $\mathrm{B}$ is to the southwest. Adopting a distance of $1.5 \mathrm{kpc}$, the radii of subshells A and B are about $7 \mathrm{pc}$ and $11 \mathrm{pc}$, respectively. Between the subshells is a darker lane that runs across the remnant from the northwest to southeast. This is a molecular cloud which has been mapped in ${ }^{12} \mathrm{CO}$ emission (Cornett et al. 1977; Dickman et al. 1992; Zhang et al. 2009), and is known to be in the foreground because it absorbs X-rays emitted by the hot remnant interior (Troja et al. 2006). Aside from this quiescent foreground cloud, observations of the $J=1 \rightarrow 0$ line of ${ }^{12} \mathrm{CO}$ also show shocked molecular material coincident with IC 443 (DeNoyer 1979; Huang et al. 1986; Dickman et al. 1992; Wang \& Scoville 1992). These shocked molecular clumps first identified by DeNoyer (1979) and Huang et al. (1986) in CO have also been observed in several atomic and small molecular species (e.g., White et al. 1987; Burton et al. 1988; van Dishoeck et al. 1993; White 1994; Snell et al. 2005) and are thought to be the result of the expanding SNR interacting with the surrounding ISM. While many of the shocked clumps are coincident with the quiescent gas, it is unclear whether or not they are part of the foreground cloud (i.e., the back portions of the foreground cloud are beginning to interact with the SNR blast wave) or if the foreground cloud is separated from IC 443.

Chemical analyses performed in various studies of the shocked clumps around IC 443 suggest an enhanced ionization rate due to cosmic rays. White (1994) found a C/CO ratio much 


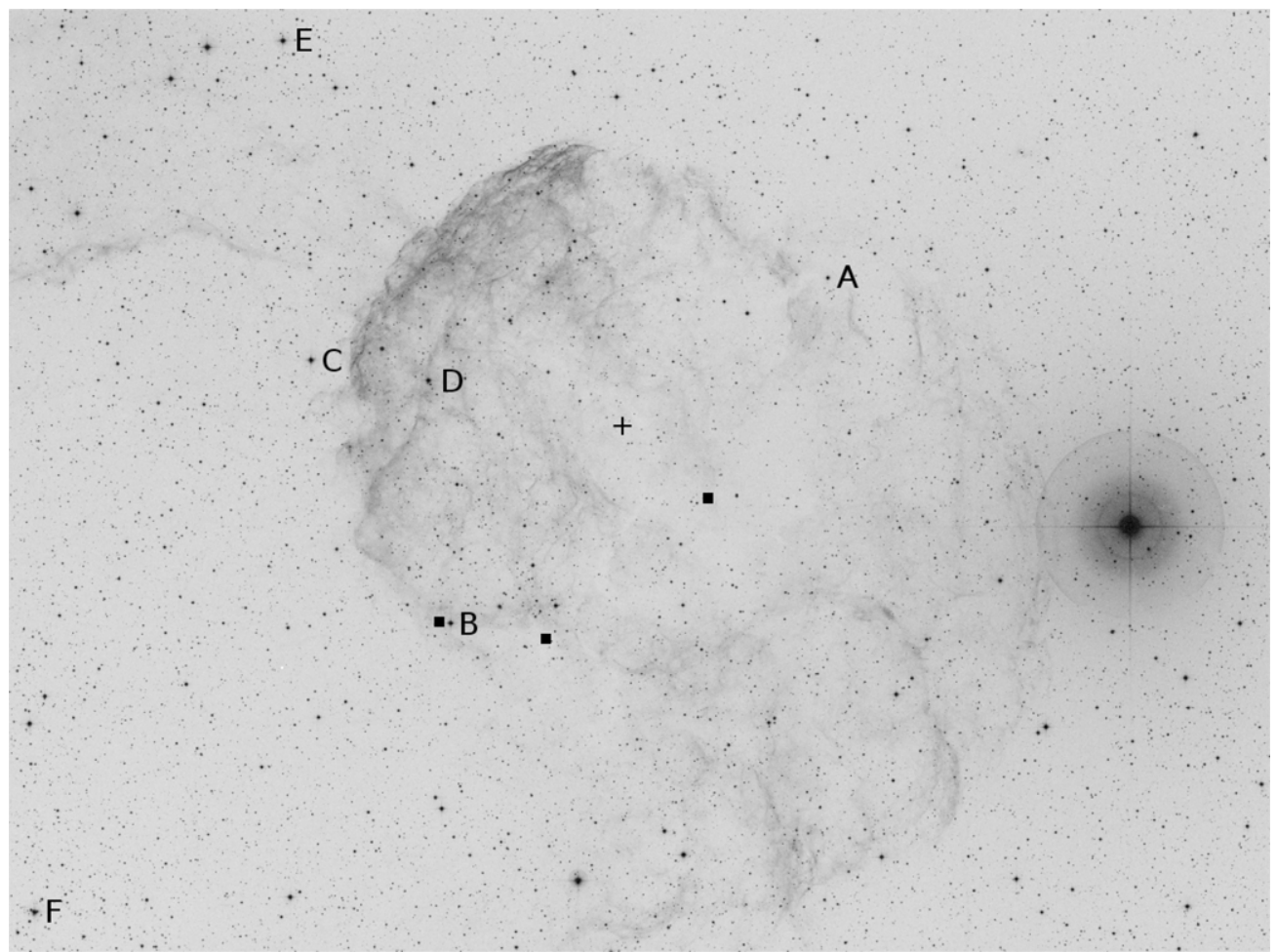

Figure 1. This image of IC 443 is from the Second Palomar Observatory Sky Survey (POSS-II) using the red filter, and was obtained from the STScI Digitized Sky Survey. Target background stars are to the immediate left of the uppercase letters, and are labeled as follows: A-ALS 8828; B-HD 254577; C-HD 254755; D-HD 43582; E-HD 43703; F-HD 43907. The cross marks the center of subshell A (the shell on the northeast side of IC 443) at $\alpha=06^{\mathrm{h}} 17^{\mathrm{m}} 08^{\mathrm{s}} 4, \delta=+22^{\circ} 36^{\prime} 39^{\prime \prime} 4 \mathrm{~J}^{\prime} 2000.0$. The three black squares mark the positions of $\mathrm{OH}(1720 \mathrm{MHz})$ maser emission reported by Hewitt et al. (2006).

higher than in typical dense clouds and concluded that shocks and/or a large flux of cosmic rays must be responsible. Both Claussen et al. (1997) and Hewitt et al. (2006) observed OH $(1720 \mathrm{MHz})$ masers toward some of these clumps. It is thought that this $\mathrm{OH}$ is formed when the free electrons produced during ionization events collide with and excite $\mathrm{H}_{2}$, which in turn emits UV photons that dissociate $\mathrm{H}_{2} \mathrm{O}$ (Wardle \& Yusef-Zadeh 2002). In order to convert nearly all of the $\mathrm{H}_{2} \mathrm{O}$ into $\mathrm{OH}$, thus generating the large column of $\mathrm{OH}$ necessary to produce the observed masers, a high ionization rate due to X-rays and/or cosmic rays is required. Estimates of the ionization rate due to X-rays (Yusef-Zadeh et al. 2003) and cosmic rays (Hewitt et al. 2009) near IC 443 are similar (a few times $10^{-16} \mathrm{~s}^{-1}$ ), so it may be that both play a role in generating $\mathrm{OH}$. However, none of these analyses alone can determine exactly how important cosmic-ray ionization and excitation are to the processes considered.

Recently, many studies of IC 443 have focused on the production of pionic gamma rays via interactions between hadronic cosmic rays and ambient nucleons. Gamma-ray observations of IC 443 have been performed by EGRET (Esposito et al. 1996), MAGIC (Albert et al. 2007), VERITAS (Acciari et al. 2009), Fermi LAT (Abdo et al. 2010), and AGILE (Tavani et al. 2010). All show gamma-ray emission that appears to be coincident with gas in the vicinity of IC 443 , thus supporting an enhanced cosmic ray flux in the region. Because $\pi^{0}$ production requires cosmic-ray protons with $E_{\text {kin }}>280 \mathrm{MeV}$, gamma-ray observations cannot constrain the cosmic-ray flux at lower energies.

To investigate the flux of lower energy cosmic rays, we study the cosmic-ray ionization of $\mathrm{H}_{2}$, a process dominated by protons with $1 \mathrm{MeV} \leqslant E_{\text {kin }} \leqslant 1 \mathrm{GeV}$ (Indriolo et al. 2009; Padovani et al. 2009). The ionization rate of $\mathrm{H}_{2}, \zeta_{2}$, can be inferred from observations of $\mathrm{H}_{3}^{+}$assuming a rather simple chemical network. $\mathrm{H}_{2}$ is first ionized, after which the ion collides with another $\mathrm{H}_{2}$, thus forming $\mathrm{H}_{3}^{+}$. Either dissociative recombination with electrons (diffuse clouds) or proton transfer to $\mathrm{CO}, \mathrm{O}$, and $\mathrm{C}$ (dense clouds) are the primary destruction routes for $\mathrm{H}_{3}^{+}$depending on the environment. In this paper, we present observations searching for absorption lines of $\mathrm{H}_{3}^{+}$along sight lines which pass through molecular material near IC 443 . We then use the results of these observations in combination with the simple chemical scheme outlined above to infer the cosmic-ray ionization rate of $\mathrm{H}_{2}$.

\section{OBSERVATIONS}

This project examined six target sight lines toward the stars ALS 8828, HD 254577, HD 254755, HD 43582, HD 43703, and HD 43907, all of which are shown in Figure 1 to the immediate left of the labels A-F, respectively. Target selection was based on various criteria, including $L$-band magnitude, previously detected molecules, and evidence that the background stars were in fact behind the SNR (Welsh \& Sallmen 2003; Hirschauer et al. 2009). Basic properties of these sight lines are available in Hirschauer et al. (2009). Observations focused primarily on transitions arising from the $(J, K)=(1,1)$ and $(1,0)$ levels of the ground vibrational state of $\mathrm{H}_{3}^{+}$, the only levels significantly populated at average diffuse cloud temperatures $(T \sim 60 \mathrm{~K})$. Transitions from higher energy levels (e.g., $(2,1)$ and $(3,3))$ were covered as allowed by the instrument, but absorption at these wavelengths was not expected.

Spectra were obtained using the Near-Infrared Echelle Spectrograph (NIRSPEC; McLean et al. 1998) at the W. M. Keck Observatory, and the Infrared Camera and Spectrograph (IRCS; Kobayashi et al. 2000) at the Subaru Telescope. All NIRSPEC observations were performed on 2009 November 5 and 6 


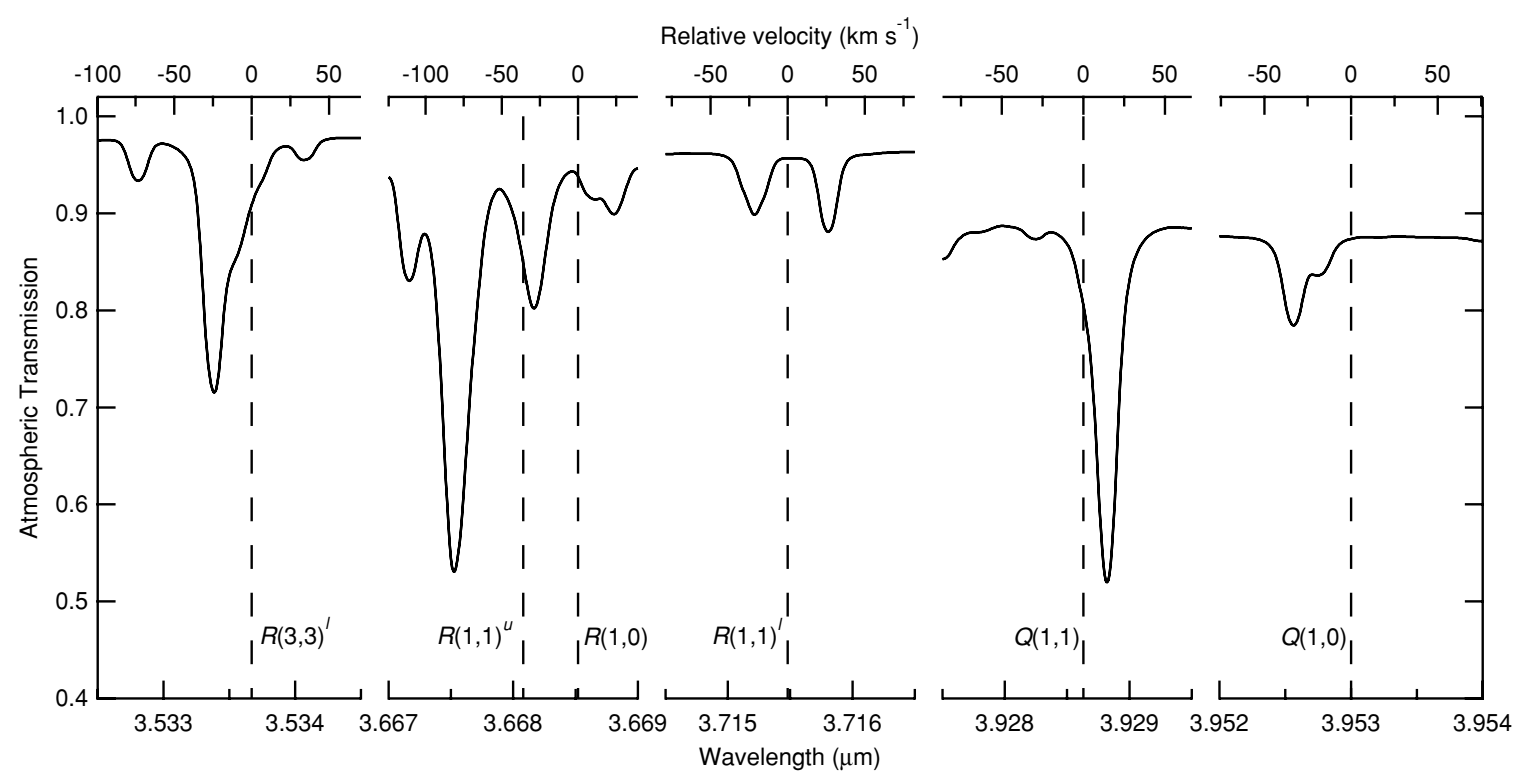

Figure 2. Model atmospheric transmission spectra at wavelengths near targeted $\mathrm{H}_{3}^{+}$transitions, generated by ATRAN (Lord 1992) assuming observations were performed from Mauna Kea, $1.6 \mathrm{~mm}$ precipitable water vapor, and an air mass of 1.15 , and smoothed to a resolving power of about 25,000 . Dashed vertical lines mark the rest positions of $\mathrm{H}_{3}^{+}$transitions, and are labeled accordingly. The bottom axes give the rest wavelengths in $\mu \mathrm{m}$, while the top axes show the effect of relative motion between the observer and interstellar gas. Average velocity shifts (due to the Earth's motion and interstellar gas motion) for the spectra taken at Keck and Subaru are about $-18 \mathrm{~km} \mathrm{~s}^{-1}$ and $-2 \mathrm{~km} \mathrm{~s}^{-1}$, respectively.

Table 1

$\mathrm{H}_{3}^{+}$Transition Properties

\begin{tabular}{lcc}
\hline \hline Transition & $\begin{array}{c}\text { Wavelength } \\
(\mu \mathrm{m})\end{array}$ & $\begin{array}{c}|\mu|^{2} \\
\left(\mathrm{D}^{2}\right)\end{array}$ \\
\hline$R(1,1)^{u}$ & 3.668083 & 0.0158 \\
$R(1,0)$ & 3.668516 & 0.0259 \\
$R(1,1)^{l}$ & 3.715479 & 0.0141 \\
$Q(1,1)$ & 3.928625 & 0.0128 \\
$Q(1,0)$ & 3.953000 & 0.0254 \\
$R(3,3)^{l}$ & 3.533666 & 0.0191 \\
\hline
\end{tabular}

Note. Wavelengths and dipole moments for targeted transitions in the $v_{2} \leftarrow 0$ band of $\mathrm{H}_{3}^{+}$(values from Goto et al. 2002 and references therein).

using the 3 pixel (0'432) slit to provide a resolving power of $\sim 25,000$. The $K L$ filter was used in combination with the echelle grating/cross-disperser settings of 64.82/33.5 in order to simultaneously cover the $R(1,1)^{u}, R(1,0)$, and $R(3,3)^{l}$ transitions. All IRCS observations were performed on 2009 December 12 and 13 in the echelle mode using the 2 pixel $\left(00^{\prime \prime} 14\right)$ slit to provide a resolving power of $\sim 17,300$. The adaptive optics system (AO188) was utilized in order to maximize starlight passing through the narrow slit. The $L$ filter was used in combination with the echelle grating/cross-disperser settings of $8350 / 6100$ in order to simultaneously cover the $R(1,1)^{u}, R(1,0), R(1,1)^{l}$, $Q(1,1)$, and $Q(1,0)$ transitions. Selected properties of the six targeted transitions are listed in Table 1, and their locations with respect to atmospheric absorption features are shown in Figure 2. A log containing the list of science targets and exposure times for each night is shown in Table 2. In addition to the science targets, the bright, early-type stars $\beta$ Tau and $\zeta$ Tau were observed for use as telluric standards. These standards were observed so that they would have good air-mass matches with the science targets, and to account for atmospheric variability over the course of each night. For all observations, the star was nodded along the slit in an ABBA pattern in order to facilitate the
Table 2

Observations

\begin{tabular}{clcc}
\hline \hline Object & Date(s) of Observation & Telescope & $\begin{array}{c}\text { Integration Time } \\
\text { (minutes) }\end{array}$ \\
\hline ALS 8828 & 2009 Nov 5 & Keck & 40 \\
& 2009 Nov 6 & Keck & 24 \\
HD 254577 & 2009 Nov 5 & Keck & 40 \\
& 2009 Nov 6 & Keck & 20 \\
& 2009 Dec 12 & Subaru & 96 \\
HD 254755 & 2009 Dec 13 & Subaru & 36 \\
& 2009 Nov 5 & Keck & 40 \\
& 2009 Nov 6 & Keck & 20 \\
HD 43582 & 2009 Dec 13 & Subaru & 120 \\
& 2009 Nov 5 & Keck & 28 \\
& 2009 Nov 6 & Keck & 40 \\
HD 43703 & 2009 Dec 12 & Subaru & 120 \\
& 2009 Nov 6 & Keck & 68 \\
HD 43907 & 2009 Dec 13 & Subaru & 120 \\
& 2009 Dec 12 & Subaru & 42 \\
\hline
\end{tabular}

removal of atmospheric emission lines and dark current via the subtraction of neighboring images.

\section{DATA REDUCTION}

Our data reduction process combines the use of standard IRAF 9 procedures and macros written in IGOR Pro..$^{10}$ Due to differences between the NIRSPEC and IRCS observations, each data set required slightly different reduction techniques.

\subsection{Keck Data Reduction Process}

A bad pixel map was created from the average of several dark frames, and these pixels were interpolated over in the flat-field and object images. Images were then cut into two

\footnotetext{
9 http://iraf.noao.edu/

10 http://www.wavemetrics.com/
} 
sections, with each section containing one of the orders of interest from the cross-dispersed spectrograph. These sections were then treated as individual images for the remainder of the reduction process. The flats were then combined, and each object frame was divided by the normalized, averaged flat field. Neighboring $A B$ image pairs were subtracted from each other to remove atmospheric emission and dark current. Onedimensional spectra were extracted for each order using apall, and imported to IGOR Pro.

\subsection{Subaru Data Reduction Process}

While no dark frames were taken at Subaru, calibration frames with the lamp off were used to create a bad pixel map. These pixels were interpolated over in the object and flat-field frames, and again each image was cut into two sections containing an order of interest. Inspection of the averaged, normalized flatfield frame for each order showed a low signal-to-noise ratio $(\mathrm{S} / \mathrm{N})$, so object frames were not divided by these flats. As before, one-dimensional spectra were extracted with apall and imported to IGOR Pro. In order to remove a saw-tooth pattern (the result of different readout channels) from these spectra, a moving average was taken for both the odd- and even-numbered pixels, and the even pixels were then scaled by the ratio of these averages.

\subsection{Shared Reduction Processes}

Individual spectra within an exposure sequence for a given target were then added together. In this process, spectra with $\mathrm{S} / \mathrm{N}$ much lower than average (due to cirrus clouds or a bad nodding sequence) were excluded. Each summed spectrum was then divided by a telluric standard to remove atmospheric absorption features and normalize the spectrum. These ratioed spectra were wavelength calibrated with a typical accuracy of $\sim 2 \mathrm{~km} \mathrm{~s}^{-1}$ using the vacuum wavelengths of the atmospheric absorption lines. Calibrated spectra were then shifted into the local standard of rest (LSR) frame. At this point all spectra of a given target from a single telescope were combined using a variance-weighted $\operatorname{mean}^{11}\left(\bar{x}=\sum_{i=0}^{n}\left(x_{i} / \sigma_{i}^{2}\right) / \sum_{i=0}^{n}\left(1 / \sigma_{i}^{2}\right)\right.$, where $\sigma_{i}$ is the standard deviation on the continuum near the $\mathrm{H}_{3}^{+}$ line positions) to produce a final Keck and Subaru spectrum for each sight line. The $R(1,1)^{l}, Q(1,1)$, and $Q(1,0)$ transitions were only covered at Subaru, while the $R(3,3)^{l}$ transition was only covered at Keck, so these are the final spectra presented in Figure 3. The $R(1,1)^{u}$ and $R(1,0)$ transitions were covered at both telescopes, so the final Keck and Subaru spectra can be combined to obtain a higher $\mathrm{S} / \mathrm{N}$. The Keck spectra were interpolated onto the lower resolution Subaru wavelength scale, and all spectra were again combined via the weighting scheme described above. The resulting spectra for our six target sight lines are shown in Figure 3.

\section{RESULTS}

It is clear from Figure 3 that $\mathrm{H}_{3}^{+}$absorption is only detected in the sight lines toward ALS 8828 and HD 254577. The $R(1,1)^{u}$ and $R(1,0)$ lines are quite strong toward ALS 8828. Absorption from the higher energy $(3,3)$ and $(2,1)$ states was not detected, as expected given diffuse molecular cloud conditions. The sight line toward HD 254577 shows absorption from the $R(1,1)^{u}$, $R(1,0), R(1,1)^{l}$ and $Q(1,0)$ transitions of $\mathrm{H}_{3}^{+}$. Absorption due to the $Q(1,1)$ transition must also be present, but it is not

\footnotetext{
11 This averaging scheme is equivalent to weighting each spectrum by $(\mathrm{S} / \mathrm{N})^{2}$.
}

detected. This is probably the result of three factors: (1) the intrinsic strength of the $Q(1,1)$ transition is the weakest of the five transitions examined (see Table 1); (2) the spectrum near the $Q(1,1)$ transition has a low $\mathrm{S} / \mathrm{N}$ due to lower illumination of the echelle order in which it appears; (3) the $Q(1,1)$ transition is overlapped by a strong atmospheric $\mathrm{N}_{2} \mathrm{O}$ line (see panel 4 of Figure 2), making removal of telluric features uncertain. Imperfect removal of this atmospheric line is also the most likely cause of the feature in the $Q(1,1)$ spectrum of HD 254755 that appears at the expected velocity. This feature cannot be due to $\mathrm{H}_{3}^{+}$, as there is no absorption by any of the other stronger $\mathrm{H}_{3}^{+}$ transitions which arise from the same state. The positive spike near $130 \mathrm{~km} \mathrm{~s}^{-1}$ in the $R(1,1)^{l}$ spectra of HD 254577 and HD 254755 is an instrumental artifact. Spectra of HD 43582, HD 43703, and HD 43907 also show no absorption features from $\mathrm{H}_{3}^{+}$.

\section{ANALYSIS}

Equivalent widths were determined using Gaussian fits to the absorption features. Uncertainties were determined from the standard deviation, $\sigma$, on the residual continuum after subtracting by the Gaussian line profiles. Interstellar gas velocities and velocity full width at half-maxima (FWHMs) were also determined during this fitting procedure. In the case of non-detections, upper limits were determined from $3 \sigma$ on the continuum across the expected position of a line assuming an FWHM of $16 \mathrm{~km} \mathrm{~s}^{-1}$ (the resolution obtained with IRCS at Subaru). Column densities were derived from equivalent widths using the standard relation given optically thin absorption lines and the transition dipole moments and wavelengths listed in Table 1. All of these results are shown in Table 3.

Following the analysis of Indriolo et al. (2007), we adopt the simple chemical scheme in diffuse clouds where every ionization of an $\mathrm{H}_{2}$ molecule leads to $\mathrm{H}_{3}^{+}$, and dissociative recombination with electrons is the dominant mechanism by which $\mathrm{H}_{3}^{+}$is destroyed. This results in the steady-state equation

$$
\zeta_{2} n\left(\mathrm{H}_{2}\right)=k_{e} n_{e} n\left(\mathrm{H}_{3}^{+}\right)
$$

(Geballe et al. 1999), where $\zeta_{2}$ is the ionization rate of $\mathrm{H}_{2}$ and $k_{e}$ is the electron recombination rate coefficient of $\mathrm{H}_{3}^{+}$. Substituting the electron fraction (defined as $x_{e} \equiv n_{e} / n_{\mathrm{H}}$, where $\left.n_{\mathrm{H}} \equiv n(\mathrm{H})+2 n\left(\mathrm{H}_{2}\right)\right)$ into Equation (1) and solving for the ionization rate gives

$$
\zeta_{2}=k_{e} x_{e} n_{\mathrm{H}} \frac{n\left(\mathrm{H}_{3}^{+}\right)}{n\left(\mathrm{H}_{2}\right)} .
$$

Although it would be desirable to trace the ionization rate as a function of position throughout the cloud, variations in density along the line of sight cannot be determined via observations. Instead, we infer the average ionization rate in a cloud by using average number densities. By definition, $\left\langle n\left(\mathrm{H}_{3}^{+}\right)\right\rangle$and $\left\langle n\left(\mathrm{H}_{2}\right)\right\rangle$ can be replaced with $N\left(\mathrm{H}_{3}^{+}\right) / L$ and $N\left(\mathrm{H}_{2}\right) / L$, respectively (where $L$ is the cloud path length), thus putting Equation (2) in terms of observables. As $\mathrm{H}_{3}^{+}$will form wherever there is an appreciable amount of $\mathrm{H}_{2}$, it is reasonable to assume that the path length for both species is the same, such that

$$
\zeta_{2}=k_{e} x_{e} n_{\mathrm{H}} \frac{N\left(\mathrm{H}_{3}^{+}\right)}{N\left(\mathrm{H}_{2}\right)} \text {. }
$$

Because the ratio $n\left(\mathrm{H}_{3}^{+}\right) / n\left(\mathrm{H}_{2}\right)$ is not expected to vary widely in models of diffuse molecular clouds (e.g., Neufeld et al. 2005), 


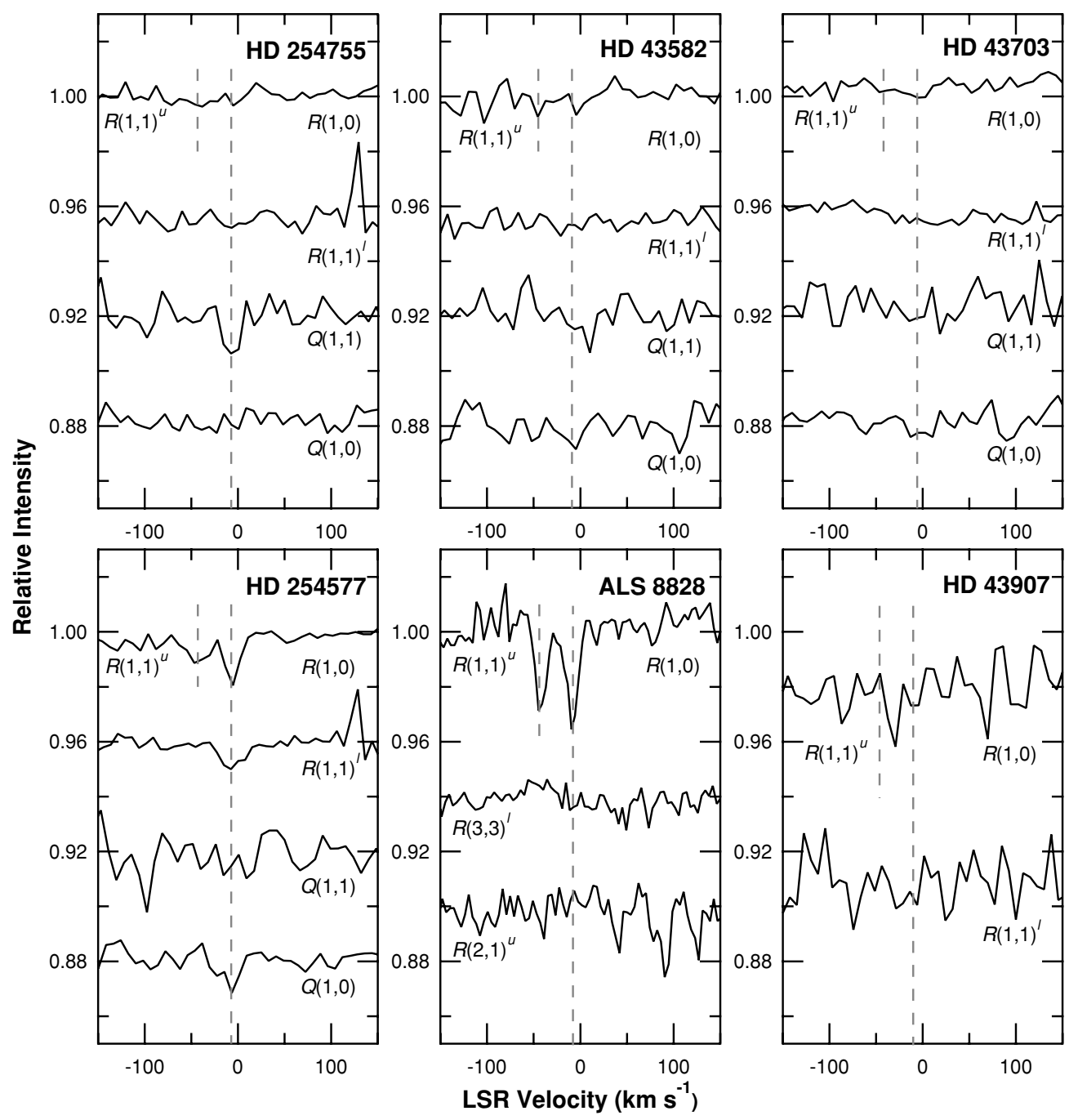

Figure 3. Spectra of six stars in the IC 443 region covering various $\mathrm{H}_{3}^{+}$transitions. Vertical dashed lines show the expected position of absorption lines due to $\mathrm{H}_{3}^{+}$given the velocities reported by Hirschauer et al. (2009) for cloud components with the most $\mathrm{CH}$ absorption. The shorter dashed line in the $R(1,1)^{u}-R(1,0)$ spectra shows the position of the $R(1,1)^{u}$ line, which is $36 \mathrm{~km} \mathrm{~s}^{-1}$ away from the $R(1,0)$ line. For HD 254755, HD 43582, HD 43703, and HD 254577, the $R(1,1)^{u}-R(1,0)$ spectra are the combination of NIRSPEC and IRCS data, while the $R(1,1)^{l}, Q(1,1)$, and $Q(1,0)$ spectra are only from IRCS. All of the ALS 8828 spectra are from NIRSPEC, and HD 43907 spectra are from IRCS. Of these six sight lines, only HD 254577 and ALS 8828 show $\mathrm{H}_{3}^{+}$absorption features. The $R(1,1)^{u}, R(1,0), R(1,1)^{l}$, and $Q(1,0)$ lines are visible toward HD 254577 (the lower S/N in the $Q(1,1)$ spectrum and smaller dipole moment of that transition precludes its detection). For ALS 8828 , relatively strong $R(1,1)^{u}$ and $R(1,0)$ lines are visible. Even with a large amount of $\mathrm{H}_{3}^{+}$along the sight line though, the $R(2,1)^{u}$ and $R(3,3)^{l}$ transitions arising from higher energy states are not detected.

this should give a representative value of the ionization rate throughout the entire cloud.

Assuming that the vast majority of electrons in diffuse molecular clouds come from photoionized carbon, $x_{e}$ can be approximated by $N\left(\mathrm{C}^{+}\right) / N_{\mathrm{H}}\left(N_{\mathrm{H}}\right.$ is the column density analog to $n_{\mathrm{H}}$ ), which was found to be about $1.5 \times 10^{-4}$ along multiple diffuse cloud sight lines (Cardelli et al. 1996; Sofia et al. 2004). The hydrogen number density can be estimated by both a rotation-excitation analysis of $\mathrm{C}_{2}$ observations and a restricted chemical analysis based on $\mathrm{CN}$ observations (Hirschauer et al. 2009). The $C_{2}$ analysis also gives a best-fit kinetic temperature which we use in calculating $k_{e}$. The temperature dependence of $k_{e}$ as determined from laboratory work is reported in McCall et al. (2004). While molecular hydrogen has not been observed in absorption along any of our target sight lines, abundances of $\mathrm{H}_{2}$ and $\mathrm{CH}$ tend to be linearly related in diffuse clouds (Federman 1982; Mattila 1986; Sheffer et al. 2008). We use the relationship derived from the largest, most recent data set- $N(\mathrm{CH}) / N\left(\mathrm{H}_{2}\right)=3.5_{-1.4}^{+2.1} \times 10^{-8}$ (Sheffer et al. 2008)-in combination with $\mathrm{CH}$ column densities reported by Hirschauer et al. (2009) to estimate $N\left(\mathrm{H}_{2}\right)$. Finally, the total $\mathrm{H}_{3}^{+}$column density is determined by adding $N(1,0)$ and $N(1,1)$. These input values and/or the parameters on which they depend, as well as the inferred ionization rates, are shown in Table 4.

While $\mathrm{H}_{2}$ is ionized by both cosmic rays and $\mathrm{X}$-rays, most of the X-ray flux should be attenuated in a relatively thin layer at the cloud exterior (Glassgold \& Langer 1974). The ionization rate due to X-rays at the edge of IC 443 was estimated to be $\zeta_{\mathrm{X}}=3.6 \times 10^{-16} \mathrm{~s}^{-1}$ (Yusef-Zadeh et al. 2003), and must be much lower in cloud interiors. As a result, the ionization rates we infer should be primarily due to cosmic rays.

\section{DISCUSSION}

Having computed the cosmic-ray ionization rate for clouds in the vicinity of IC 443, we compare our results to those from previous studies. The average ionization rate in diffuse molecular clouds found by Indriolo et al. (2007) using $\mathrm{H}_{3}^{+}$ 
Table 3

Absorption Line Parameters

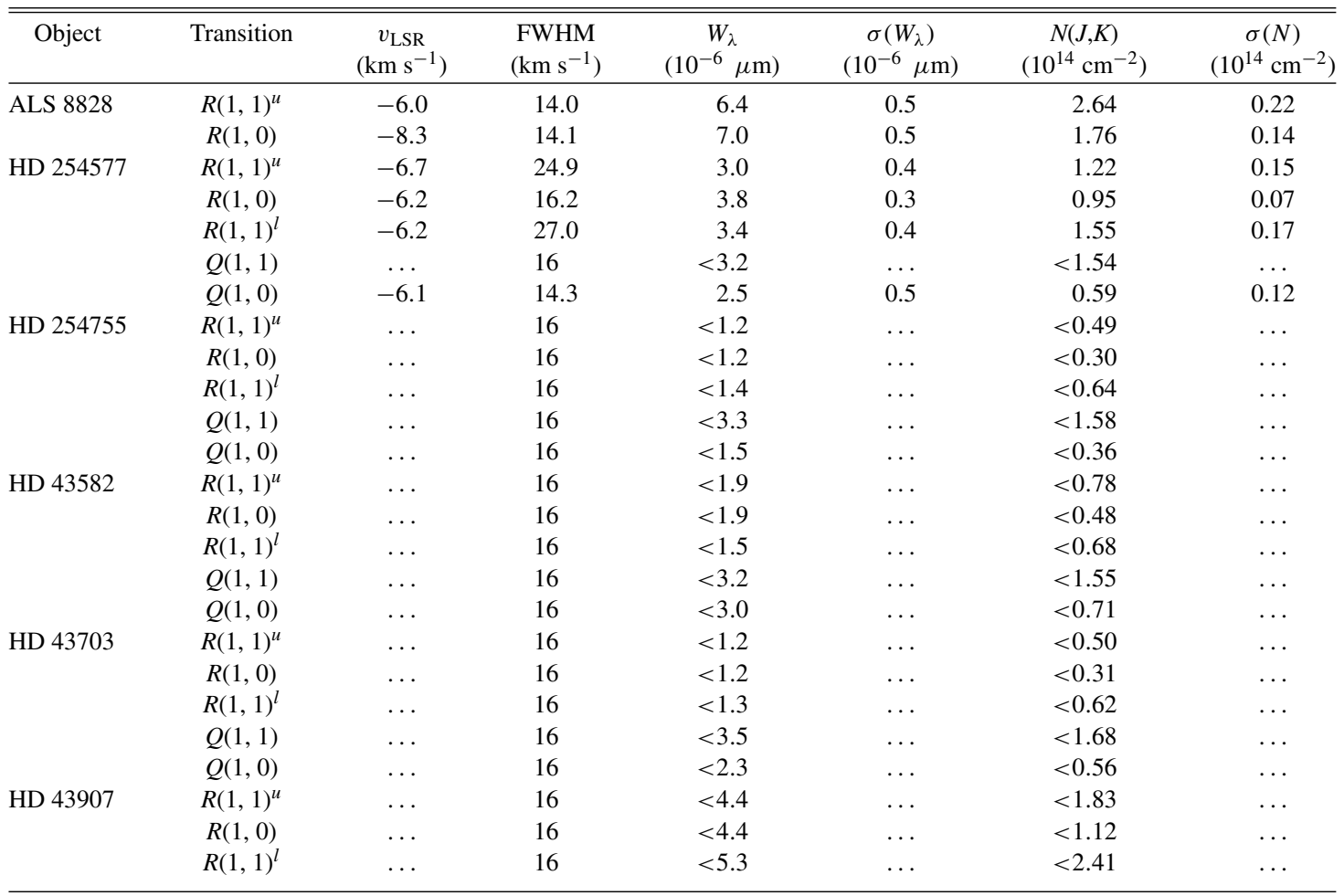

Notes. Column $3\left(v_{\text {LSR }}\right)$ gives the interstellar gas velocity in the LSR frame. Column 4 gives the FWHM of the absorption features. In the case of non-detections, the FWHM was set to $16 \mathrm{~km} \mathrm{~s}^{-1}$, the resolving power of IRCS on Subaru in our particular setup, for the purpose of computing column density upper limits. Columns 5 and 6 show the equivalent width, $W_{\lambda}$, and its $1 \sigma$ uncertainty, $\sigma\left(W_{\lambda}\right)$, respectively. Upper limits to $W_{\lambda}$ are equal to $3 \sigma\left(W_{\lambda}\right)$. Columns 7 and 8 give the column density of $\mathrm{H}_{3}^{+}$in the state each transition probes, $N(J, K)$, and its uncertainty, $\sigma(N)$, respectively. Upper limits to the $\mathrm{H}_{3}^{+}$column density are equal to $3 \sigma(N)$.

Table 4

Target Sight Line Properties

\begin{tabular}{|c|c|c|c|c|c|c|c|c|}
\hline Target & $\begin{array}{c}r \\
(\mathrm{pc})\end{array}$ & $\begin{array}{c}T \\
(\mathrm{~K})\end{array}$ & $\begin{array}{c}n_{\mathrm{H}} \\
\left(\mathrm{cm}^{-3}\right)\end{array}$ & $\begin{array}{c}N_{\mathrm{H}} \\
\left(10^{21} \mathrm{~cm}^{-2}\right)\end{array}$ & $\begin{array}{c}L \\
(\mathrm{pc})\end{array}$ & $\begin{array}{c}N\left(\mathrm{H}_{2}\right) \\
\left(10^{21} \mathrm{~cm}^{-2}\right)\end{array}$ & $\begin{array}{c}N\left(\mathrm{H}_{3}^{+}\right) \\
\left(10^{14} \mathrm{~cm}^{-2}\right)\end{array}$ & $\begin{array}{c}\zeta_{2} \\
\left(10^{-16} \mathrm{~s}^{-1}\right)\end{array}$ \\
\hline ALS 8828 & $6.8^{\mathrm{a}}$ & $60^{b}$ & $300^{c}$ & 3.0 & 3.2 & $2.1_{-0.8}^{+1.4}$ & $4.4 \pm 0.26$ & $16_{-12}^{+8}$ \\
\hline HD 254755 & 8.6 & $35^{\mathrm{d}}$ & $200^{d}$ & 2.5 & 4.0 & $1.1_{-0.4}^{+0.7}$ & $<0.6$ & $<3.5$ \\
\hline HD 43582 & $5.4^{\mathrm{a}}$ & $60^{\mathrm{b}}$ & $200^{\mathrm{e}}$ & 1.9 & 3.0 & $0.5_{-0.2}^{+0.3}$ & $<0.8$ & $<9.0$ \\
\hline HD 43907 & 20.6 & $60^{\mathrm{b}}$ & $300^{c}$ & 1.5 & 1.6 & $0.4_{-0.2}^{+0.3}$ & $<2.1$ & $<40$ \\
\hline
\end{tabular}

Notes. Various parameters used in our analysis for the target sight lines in this study. Column 2 gives the on-sky distance $(r)$ from the center of shell A of IC $443\left(\alpha=06^{\mathrm{h}} 17^{\mathrm{m}} 08^{\mathrm{s}} .4, \delta=+22^{\circ} 36^{\prime} 39^{\prime \prime} .4 \mathrm{~J} 2000.0\right)$ to each sight line assuming the remnant is at a distance of $1.5 \mathrm{kpc}$. The radius of shell $\mathrm{A}$ is about $7 \mathrm{pc}$. Temperatures $(T)$ were taken from the $\mathrm{C}_{2}$ rotation-excitation analysis when available or set to $60 \mathrm{~K}$, and number densities $\left(n_{\mathrm{H}}\right)$ were taken from either the $\mathrm{C}_{2}$ rotation-excitation analysis or restricted chemical analysis, both reported in Hirschauer et al. (2009). Uncertainties in $n_{\mathrm{H}}$ are taken to be $\pm 100 \mathrm{~cm}^{-3}$. Color excesses were decreased by 0.3 mag to remove the contribution from foreground gas (color excesses and the foreground correction are given in Hirschauer et al. 2009), and the relationship $N_{\mathrm{H}} \approx E(B-V) \times 5.8 \times 10^{21} \mathrm{~cm}^{-2} \mathrm{mag}^{-1}$ (Bohlin et al. 1978; Rachford et al. 2002) was used to compute the total hydrogen column densities, $N_{\mathrm{H}}$. Path lengths $(L)$ were calculated from $n_{\mathrm{H}}$ and $N_{\mathrm{H}}$. Molecular hydrogen column densities were calculated from the relationship $N(\mathrm{CH}) / N\left(\mathrm{H}_{2}\right)=3.5_{-1.4}^{+2.1} \times 10^{-8}$ (Sheffer et al. 2008) using the dominant $\mathrm{CH}$ components from Hirschauer et al. (2009). Uncertainties in $N\left(\mathrm{H}_{2}\right)$ are dominated by the scatter in the above relationship, not uncertainties in $N(\mathrm{CH})$. Upper limits for $N\left(\mathrm{H}_{3}^{+}\right)$are the $3 \sigma$ uncertainties from the observations, and the upper limits for $\zeta_{2}$ are based solely on those values. To account for the uncertainty in $N\left(\mathrm{H}_{2}\right)$, the upper limits for $\zeta_{2}$ should be multiplied by 1.5 .

a These sight lines pass through the remnant.

${ }^{\mathrm{b}}$ Kinetic temperature assumed in restricted chemical analysis.

${ }^{c}$ Average density from restricted chemical analysis.

${ }^{\mathrm{d}}$ Determined from $\mathrm{C}_{2}$ rotation-excitation analysis.

e As no CN was detected toward HD 43582, here we have taken the lower bound on the density from the restricted chemical analysis for other sight lines. 
was $\zeta_{2}=4 \times 10^{-16} \mathrm{~s}^{-1}$, several times lower than that found toward ALS 8828 and HD $254577\left(\zeta_{2}=16_{-12}^{+8} \times\right.$ $10^{-16} \mathrm{~s}^{-1}$ and $26_{-19}^{+13} \times 10^{-16} \mathrm{~s}^{-1}$, respectively). In fact, the ionization rates inferred for these two sight lines are more than twice the highest rates previously found in diffuse molecular clouds toward $\zeta$ Per and X Per $\left(\zeta_{2} \approx 7 \times 10^{-16} \mathrm{~s}^{-1}\right)$. While ALS 8828 and HD 254577 present exceptionally high ionization rates, the other three sight lines observed near IC 443 do not (due to the low S/N obtained toward HD 43907, the derived upper limit for that particular sight line is not exceptionally meaningful, and so we exclude it from further consideration). Instead, the $3 \sigma$ upper limits for $\zeta_{2}$ presented in Table 4 are consistent with ionization rates of a few times $10^{-16} \mathrm{~s}^{-1}$, typical of diffuse molecular clouds. These differences are quite striking, and warrant discussion.

There are two plausible explanations for why $\mathrm{H}_{3}^{+}$would be detected toward ALS 8828 and HD 254577 but not toward HD 254755, HD 43582, HD 43703, and HD 43907, and they can most easily be seen when Equation (3) is rearranged to show that $N\left(\mathrm{H}_{3}^{+}\right) \propto \zeta_{2} /\left(x_{e} n_{\mathrm{H}}\right)$. Given this scaling, we can posit that either the product $x_{e} n_{\mathrm{H}}$ (i.e., the electron density) is lower along these two sight lines, or $\zeta_{2}$ is higher, and we examine these possibilities in turn.

\subsection{Lower Electron Density}

As stated in Section 5, we have assumed an electron fraction that is consistent with observations of $\mathrm{C}^{+}$in several diffuse molecular clouds. In denser environments though, the predominant form of carbon shifts from $\mathrm{C}^{+}$to $\mathrm{C}$, and eventually to $\mathrm{CO}$, thus decreasing the electron density. Adopting a reduced value for $x_{e} n_{\mathrm{H}}$ requires a corresponding decrease in $\zeta_{2}$ to match the observed $\mathrm{H}_{3}^{+}$column density. It could then be argued that the enhanced ionization rate we calculate for the two sight lines where we detect $\mathrm{H}_{3}^{+}$is actually just an artifact of not recognizing a decreased destruction rate.

However, there are observations which seem to argue against this possibility. The $\mathrm{C}_{2}$ rotation-excitation and $\mathrm{CN}$ restricted chemical analyses performed by Hirschauer et al. (2009) suggest densities of $200-400 \mathrm{~cm}^{-3}$, typical of diffuse molecular clouds, not dense clouds. Also, we can estimate the fractional abundance of $\mathrm{CO}\left(x(\mathrm{CO})=N(\mathrm{CO}) / N_{\mathrm{H}}\right)$ in the observed sight lines and compare it to the solar system abundance of carbon $\left(x\left(\mathrm{C}_{\mathrm{tot}}\right)=\right.$ $2.9 \times 10^{-4}$; Lodders 2003) to determine if $\mathrm{CO}$ is the dominant carbon-bearing species. We estimate $N_{\mathrm{H}}$ from the color excess (see Table 4), and use observed relationships between $N(\mathrm{CH})$, $N(\mathrm{CN})$, and $N(\mathrm{CO})$ (Sonnentrucker et al. 2007; Sheffer et al. 2008), in concert with $\mathrm{CH}$ and $\mathrm{CN}$ column densities (Hirschauer et al. 2009) to estimate $N(\mathrm{CO})$. In the ALS 8828 and HD 254577 sight lines, $x(\mathrm{CO}) \sim 5 \times 10^{-6}$ and $2 \times 10^{-6}$, respectively, much smaller than the assumed total carbon budget. In the other three sight lines, $x(\mathrm{CO})$ ranges from about $1 \times 10^{-7}$ to about $1 \times 10^{-6}$. These estimates show that most carbon is not in the form of $\mathrm{CO}$, but does not rule out $\mathrm{C}$ as the dominant carbon-bearing species. To do so, we use the observed relationship between $\mathrm{CO} / \mathrm{H}_{2}$ and $(\mathrm{C}+\mathrm{CO}) / \mathrm{C}_{\text {tot }}$ shown in Figure 6 of Burgh et al. (2010). For $\mathrm{CO} / \mathrm{H}_{2} \sim 8 \times 10^{-6}$ - the largest value estimated along any of our sight lines-observations show that both $\mathrm{CO}$ and $\mathrm{C}$ account for only a small fraction of the total carbon budget, thus indicating that carbon is predominantly in ionized form.

To improve upon these rough arguments though, observations yielding the relative abundances of $\mathrm{C}^{+}, \mathrm{C}$, and $\mathrm{CO}$ are necessary. The $v=1-0$ fundamental and $v=2-0$ overtone rovibrational bands of $\mathrm{CO}$ near $4.6 \mu \mathrm{m}$ and $2.3 \mu \mathrm{m}$, respectively, can be observed with NIRSPEC and IRCS. Various electronic transitions of $\mathrm{CO}$ and $\mathrm{C}_{\mathrm{I}}$ are available in the far ultraviolet (1100 $\AA-1700 \AA)$, and can be observed with either COS or STIS aboard Hubble. Finally, a weak intersystem line of C II is at $2325 \AA$, and may also be observable with COS and/or STIS. Combined, these observations would allow us to determine the predominant carbon-bearing species along each sight line, and give us a better understanding of cloud conditions being probed.

\subsection{Higher Ionization Rate}

If the gas conditions in all of our observed sight lines are similar, then the cosmic-ray ionization rate must be higher toward ALS 8828 and HD 254577. Such varied ionization rates can be the result of differing cosmic-ray fluxes in each sight line. If we assume that the SNR accelerates particles isotropically (i.e., the spectrum of cosmic rays leaving the remnant is identical everywhere along the blast wave), then the different cosmic-ray spectra operating in each sight line must be due to propagation effects.

To determine whether or not cosmic rays accelerated by IC 443 can even produce the high inferred ionization rates, we use the methods described in Indriolo et al. (2009) to compute the expected ionization rate for various cosmic-ray spectra. Abdo et al. (2010), Torres et al. (2008), and Torres et al. (2010) constrain the proton spectrum above $\sim 100 \mathrm{MeV}$ near IC 443 from the observed gamma-ray spectrum. Although the broken power-law proton spectrum in Abdo et al. (2010) is given as a power law in kinetic energy (flux $\propto E_{\text {kin }}^{-2.09}$ when $\left.E_{\text {kin }}<69 \mathrm{GeV}\right)$, we change this to a power law in momentum (flux $\propto p^{-2.09}$, where $\left.p c=\left[\left(E_{\mathrm{kin}}+m_{p} c^{2}\right)^{2}-\left(m_{p} c^{2}\right)^{2}\right]^{0.5}\right)$ to account for the fact that diffusive shock acceleration is expected to produce a spectrum of this form. This substitution only differs from the relation considered by Abdo et al. (2010) in the nonrelativistic regime where $p \propto E_{\mathrm{kin}}^{0.5}$. As we extrapolate the spectrum to lower energies, the particle distribution is flattened relative to a pure power law in kinetic energy.

Integrating this extrapolated spectrum to a low-energy cutoff of $5 \mathrm{MeV}$, we find $\zeta_{2} \sim 10^{-14} \mathrm{~s}^{-1}$. A $5 \mathrm{MeV}$ cutoff was used because particles of this energy have a range of a few times $10^{21} \mathrm{~cm}^{-2}$ (Padovani et al. 2009), similar to the sight lines we consider here. Using the $d=10 \mathrm{pc}$ continuous injection spectrum (also extrapolated to low energies as above) from Torres et al. (2008) produces similar results, while their $d=30 \mathrm{pc}$ spectrum fails to reproduce even the ionization rate predicted by the local interstellar cosmic-ray spectrum $\left(\zeta_{2} \sim 4 \times 10^{-17} \mathrm{~s}^{-1}\right.$; Webber 1998). Note that the difference between the 10 and $30 \mathrm{pc}$ theoretical spectra is not the result of energy losses, but due to the fact that lower energy particles have not yet had sufficient time to travel far from IC 443 given its age of 30,000 yr. Although these spectra are not particularly well suited for estimating the cosmic-ray ionization rate-they are based on observations which depend on processes requiring $E_{\text {kin }}>280 \mathrm{MeV}$ and so are not well constrained at energies of a few $\mathrm{MeV}$ where ionization is much more efficient-they do suggest that cosmic rays accelerated by IC 443 are capable of generating the ionization rate inferred from $\mathrm{H}_{3}^{+}$, and also provide independent constraints on the flux of high-energy cosmic rays near IC 443 which complement the low-energy component studied in this paper.

The propagation effects included in the model cosmic-ray spectra presented in Torres et al. $(2008,2010)$ may also be able to explain the differences inferred in $\zeta_{2}$. Those authors 
suggest that the difference in centroid position between the GeV (EGRET and Fermi LAT) and TeV (MAGIC and VERITAS) gamma-ray sources can be explained by having the gamma rays of different energies originate in separate clouds. They propose that the lower energy gamma rays arise from $\pi^{0}$ decay in a cloud 3-6 pc away from the expanding shell of IC 443, and the higher energy gamma rays in a cloud about $10 \mathrm{pc}$ in front of the SNR. This explanation requires that cosmic-ray propagation is energy dependent, such that high-energy particles have diffused farther away from the SNR than low-energy particles. In such a model, the cosmic-ray spectrum varies as a function of position, and so the ionization rate must as well.

Because lower energy cosmic rays have yet to propagate very far from IC 443 , the ionization rate should decrease with increased distance away from the SNR. If the clouds probed by ALS 8828 and HD 254577 are closer to IC 443 than the clouds probed by the other three sight lines, then the difference in inferred ionization rates is easily explained. The positions of our target sight lines with respect to IC 443 are shown in Figure 1, and the on-sky distances from the center of subshell A to each of the background stars are listed in Table 4 . Of the five sight lines, only HD 43703 is a considerable distance away from the remnant, so differences in the remaining four sight lines must be due to line-of-sight distances. Gas velocities for the dominant $\mathrm{CH}$ components reported by Hirschauer et al. (2009) vary by only about $3 \mathrm{~km} \mathrm{~s}^{-1}$ between all of our sight lines, suggesting that the absorption may arise from the same cloud complex, but because IC 443 is located near the Galactic anti-center such an analysis is highly uncertain. The HD 254577 sight line passes through regions of $\mathrm{HCO}^{+}$emission (Dickman et al. 1992) and $\mathrm{H}_{2}$ emission (Burton et al. 1988; Inoue et al. 1993; Rho et al. 2001), both of which trace shocked gas, and is in close proximity to an $\mathrm{OH}(1720 \mathrm{MHz})$ maser which requires shocked gas and a high ionization rate (Hewitt et al. 2006), so it is plausible that the observed $\mathrm{H}_{3}^{+}$absorption arises in material very close to the SNR shock. The ALS 8828 sight line, however, is not coincident with shock tracers, so it is unclear at this location how close the foreground cloud is to the SNR. Still, given the drastic difference in the $10 \mathrm{pc}$ and $30 \mathrm{pc}$ cosmic-ray spectra from Torres et al. (2008, see their Figure 1), the gas probed by the three sight lines without observed $\mathrm{H}_{3}^{+}$would not have to be that much farther away than the gas probed by ALS 8828 to explain the inferred ionization rates; something on the order of $10 \mathrm{pc}$ farther away would suffice.

Aside from the distance between the site of particle acceleration and the clouds in question, various other propagation and acceleration effects could account for the difference in inferred ionization rates. Cosmic rays diffuse through space as they scatter off of Alfvén waves which are presumed to be generated by the particles themselves. For clouds with higher densities of neutral gas, the damping of these waves (via ion-neutral collisions) becomes more efficient and the streaming velocity of cosmic rays thus increases (Padoan \& Scalo 2005). Instead of diffusing then, particles will free stream and spend much less time in the cloud (i.e., have fewer chances to ionize ambient material). As a result, regions of low gas density should be expected to have higher ionization rates than regions of high gas density. Another possibility is that the net flux of cosmic rays into a cloud (due to ionization losses, nuclear interactions, etc. within the cloud) sets up an anisotropy that causes the growth of Alfvén waves in the plasma surrounding the cloud. Lower energy particles $\left(E_{\text {kin }}\right.$ less than a few hundred $\mathrm{MeV}$ ) scatter off of these waves and are impeded from entering the cloud (Skilling \& Strong
1976). Because the particles most efficient at ionizing hydrogen are excluded from denser clouds, this effect also predicts a higher ionization rate in regions of lower density. However, due to the similar densities reported in Hirschauer et al. (2009) for our target sight lines, these effects seem unlikely candidates for causing the difference in inferred ionization rates.

The final effect we consider in attempting to explain these variations in the ionization rate is the escape of cosmic rays upstream from the shock where diffusive shock acceleration occurs (i.e., away from the SNR). This subject has been the focus of several recent studies (e.g., Caprioli et al. 2009, 2010; Reville et al. 2009; Ohira et al. 2010) which find that particles can escape in the upstream direction, although these tend to be only the particles with the highest energies. For the discussion above, we have assumed that low-energy cosmic rays have escaped from the shock and are diffusing away from the SNR. However, if lowenergy cosmic rays do not escape, but are instead preferentially advected downstream (i.e., into the SNR), then the ionization rate in the post-shock gas inside the SNR should be higher than in the gas exterior to the remnant. The differing ionization rates could then be explained if the sight lines toward ALS 8828 and HD 254577 probed gas interior to IC 443. As mentioned above, the HD 254577 sight line is coincident with various shock tracers, as well as an $\mathrm{OH}(1720 \mathrm{MHz})$ maser (which arises from the post-shock gas inside the SNR). Additionally, the velocity of this maser, $-6.85 \mathrm{~km} \mathrm{~s}^{-1}$, is consistent with the $\mathrm{H}_{3}^{+}$velocities reported in Table 3, making it highly plausible that the $\mathrm{H}_{3}^{+}$absorption toward HD 254577 arises from shocked gas inside of IC 443. Consequently, the inability of low-energy cosmic rays to escape from IC 443 provides an alternative to the diffusion of particles and differing distances between the remnant and gas probed by our sight lines in explaining the inferred ionization rates.

\subsection{Implications}

Given either of the cases discussed above (low electron density or high ionization rate), we can comment on the flux of low-energy cosmic rays accelerated by SNRs. In the case that the exceptional $\mathrm{H}_{3}^{+}$column densities observed are due to a lower destruction rate (i.e., lower electron density), then the ionization rate near IC 443 is no higher than already found toward various diffuse molecular cloud sight lines. This would indicate that the flux of low-energy cosmic rays near SNRs is not substantially different than in the Galactic ISM, and suggest that either SNRs are not the primary accelerators of such particles, or that lowenergy particles have yet to escape from IC 443.

In the case that the inferred ionization rates of a few times $10^{-15} \mathrm{~s}^{-1}$ are correct, IC 443 must be accelerating a large population of low-energy cosmic rays. Either this population must be escaping upstream from the site of diffusive shock acceleration (i.e., traveling outward from the SNR shock) such that the clouds closest to the remnant are experiencing a large flux of cosmic rays, or the two sight lines with $\mathrm{H}_{3}^{+}$detections probe gas inside of IC 443 where low-energy cosmic rays have been advected downstream. In either situation, it is unclear if such a population of cosmic rays accelerated by all SNRs within the Galaxy will propagate far enough from their sources to affect the flux of cosmic rays at some arbitrary position. As a result, it is difficult to definitively say whether or not SNRs are responsible for accelerating the large flux of low-energy cosmic rays necessary to produce the $\zeta_{2} \sim 4 \times 10^{-16} \mathrm{~s}^{-1}$ ionization rate inferred in many diffuse Galactic sight lines. 


\section{CONCLUSIONS}

We have searched for $\mathrm{H}_{3}^{+}$absorption along six sight lines that pass through molecular material in the vicinity of the SNR IC 443. Two of the observed sight lines, ALS 8828 and HD 254577, have large column densities of $\mathrm{H}_{3}^{+}$, while the other four show no absorption features. The cosmic-ray ionization rates inferred from the two detections are a few times $10^{-15} \mathrm{~s}^{-1}$, higher than ever previously found in diffuse molecular clouds. Upper limits to the ionization rate in the other sight lines, however, are consistent with values found along average Galactic sight lines of about $4 \times 10^{-16} \mathrm{~s}^{-1}$. These differences may be due to overestimates of the electron fraction or cosmic-ray propagation and acceleration effects, but the complexity of the region makes it difficult to attribute the results to any one cause. Future observations of $\mathrm{C} \mathrm{II,} \mathrm{C} \mathrm{I,} \mathrm{and} \mathrm{CO} \mathrm{toward} \mathrm{our} \mathrm{target} \mathrm{sight}$ lines should allow us to better discriminate between the two possibilities, and thus determine whether or not IC 443 produces a large flux of low-energy cosmic rays.

In addition, surveys of $\mathrm{H}_{3}^{+}$near IC 443 and other SNRs thought to be interacting with molecular clouds (e.g., Vela, W 28, W 44, W 51C) should allow us to further investigate cosmic-ray acceleration in such environments. By more extensively mapping $\mathrm{H}_{3}^{+}$absorption near SNRs, we can determine where $\mathrm{H}_{3}^{+}$resides (interior post-shock gas or exterior pre-shock gas), and thus where the flux of low-energy cosmic rays is highest. Such observations may also provide insight into the efficiency with which accelerated particles are advected downstream into remnants, and so add important constraints to models of cosmic-ray acceleration.

The authors thank Steve Federman, Farhad Yusef-Zadeh, and the anonymous referee for helpful comments and suggestions. N.I. and B.J.M. are supported by NSF grant PHY 08-55633. G.A.B. is supported by NSF grant AST 07-08922. T.O. is supported by NSF grant AST 08-49577. T.R.G.'s research is supported by the Gemini Observatory, which is operated by the Association of Universities for Research in Astronomy, Inc., on behalf of the international Gemini partnership of Argentina, Australia, Brazil, Canada, Chile, the United Kingdom, and the United States of America. The work of B.D.F. was partially supported by the NASA Astrophysics Theory Program through award NNX10AC86G. The Digitized Sky Surveys were produced at the Space Telescope Science Institute under U.S. Government grant NAG W-2166. The images of these surveys are based on photographic data obtained using the Oschin Schmidt Telescope on Palomar Mountain and the UK Schmidt Telescope. The plates were processed into the present compressed digital form with the permission of these institutions. The Second Palomar Observatory Sky Survey (POSS-II) was made by the California Institute of Technology with funds from the National Science Foundation, the National Geographic Society, the Sloan Foundation, the Samuel Oschin Foundation, and the Eastman Kodak Corporation.

\section{REFERENCES}

Abdo, A. A., et al. 2010, ApJ, 712, 459

Acciari, V. A., et al. 2009, ApJ, 698, L133
Albert, J., et al. 2007, ApJ, 664, L87

Blandford, R., \& Eichler, D. 1987, Phys. Rep., 154, 1

Bohlin, R. C., Savage, B. D., \& Drake, J. F. 1978, ApJ, 224, 132

Burgh, E. B., France, K., \& Jenkins, E. B. 2010, ApJ, 708, 334

Burton, M. G., Geballe, T. R., Brand, P. W. J. L., \& Webster, A. S. 1988, MNRAS, 231, 617

Caprioli, D., Amato, E., \& Blasi, P. 2010, Astropart. Phys., 33, 307

Caprioli, D., Blasi, P., \& Amato, E. 2009, MNRAS, 369, 2065

Cardelli, J. A., Meyer, D. M., Jura, M., \& Savage, B. D. 1996, ApJ, 467, 334

Chevalier, R. A. 1999, ApJ, 511, 798

Claussen, M. J., Frail, D. A., Goss, W. M., \& Gaume, R. A. 1997, ApJ, 489, 143

Cornett, R. H., Chin, G., \& Knapp, G. R. 1977, A\&A, 54, 889

DeNoyer, L. K. 1979, ApJ, 232, L165

Dickman, R. L., Snell, R. L., Ziurys, L. M., \& Huang, Y.-L. 1992, ApJ, 400, 203

Drury, L. O’C. 1983, Rep. Prog. Phys., 46, 973

Esposito, J. A., Hunter, S. D., Kanbach, G., \& Sreekumar, P. 1996, ApJ, 461, 820

Federman, S. R. 1982, ApJ, 257, 125

Geballe, T. R., McCall, B. J., Hinkle, K. H., \& Oka, T. 1999, ApJ, 510, 251

Glassgold, A. E., \& Langer, W. D. 1974, ApJ, 193, 73

Goto, M., McCall, B. J., Geballe, T. R., Usuda, T., Kobayashi, N., Terada, H., \& Oka, T. 2002, PASJ, 54, 951

Hewitt, J. W., Yusef-Zadeh, F., \& Wardle, M. 2009, ApJ, 706, L270

Hewitt, J. W., Yusef-Zadeh, F., Wardle, M., Roberts, D. A., \& Kassim, N. E. 2006, ApJ, 652, 1288

Hirschauer, A., Federman, S. R., Wallerstein, G., \& Means, T. 2009, ApJ, 696, 1533

Huang, Y.-L., Dickman, R. L., \& Snell, R. L. 1986, ApJ, 302, L63

Indriolo, N., Fields, B. D., \& McCall, B. J. 2009, ApJ, 694, 257

Indriolo, N., Geballe, T. R., Oka, T., \& McCall, B. J. 2007, ApJ, 671, 1736

Inoue, M. Y., et al. 1993, PASJ, 45, 539

Kobayashi, N., et al. 2000, Proc. SPIE, 4008, 1056

Lodders, K. 2003, ApJ, 591, 1220

Lord, S. D. 1992, NASA Technical Memorandum 103957

Mattila, K. 1986, A\&A, 160, 157

McCall, B. J., et al. 2004, Phys. Rev. A, 70, 052716

McLean, I. S., et al. 1998, Proc. SPIE, 3354, 566

Neufeld, D. A., Wolfire, M. G., \& Schilke, P. 2005, ApJ, 628, 260

Ohira, Y., Murase, K., \& Yamazaki, R. 2010, A\&A, 513, A17

Padoan, P., \& Scalo, J. 2005, ApJ, 624, L97

Padovani, M., Galli, D., \& Glassgold, A. E. 2009, A\&A, 501, 619

Rachford, B. L., et al. 2002, ApJ, 577, 221

Reville, B., Kirk, J. G., \& Duffy, P. 2009, ApJ, 694, 951

Rho, J., Jarrett, T. H., Cutri, R. M., \& Reach, W. T. 2001, ApJ, 547, 885

Sheffer, Y., Rogers, M., Federman, S. R., Abel, N. P., Gredel, R., Lambert, D. L., \& Shaw, G. 2008, ApJ, 687, 1075

Skilling, J., \& Strong, A. W. 1976, A\&A, 53, 253

Snell, R. L., Hollenbach, D., Howe, J. E., Neufeld, D. A., Kaufman, M. J., Melnick, G. J., Bergin, E. A., \& Wang, Z. 2005, ApJ, 620, 758

Sofia, U. J., Lauroesch, J. T., Meyer, D. M., \& Cartledge, S. I. B. 2004, ApJ, 605,272

Sonnentrucker, P., Welty, D. E., Thorburn, J. A., \& York, D. G. 2007, ApJS, 168,58

Tavani, M., et al. 2010, ApJ, 710, L151

Torres, D. F., Rodriguez Marrero, A. Y., \& de Cea del Pozo, E. 2008, MNRAS, 387, L59

Torres, D. F., Rodriguez Marrero, A. Y., \& de Cea del Pozo, E. 2010, MNRAS, 408, 1257

Troja, E., Bocchino, F., \& Reale, F. 2006, ApJ, 649, 258

van Dishoeck, E. F., Jansen, D. J., \& Phillips, T. G. 1993, A\&A, 279, 541

Wang, Z., \& Scoville, N. Z. 1992, ApJ, 386, 158

Wardle, M., \& Yusef-Zadeh, F. 2002, Science, 296, 2350

Webber, W. R. 1998, ApJ, 506, 329

Welsh, B. Y., \& Sallmen, S. 2003, A\&A, 408, 545

White, G. J. 1994, A\&A, 283, L25

White, G. J., Rainey, R., Hayashi, S. S., \& Kaifu, N. 1987, A\&A, 173, 337

Yusef-Zadeh, F., Wardle, M., Rho, J., \& Sakano, M. 2003, ApJ, 585, 319

Zhang, Z., Gao, Y., \& Wang, J. 2009, Sci. China Ser. G, in press (arXiv:0911.4815v2) 